\title{
Laboreal
}

Volume 15 N$^{\circ} 2$ | 2019

Varia

\section{Clínicas del trabajo en Chile : tres experiencias de intervención en una sociedad dañada por la gestión 'managerial'}

Clínicas do trabalho no Chile : três experiências de intervenção em uma sociedade danificada por uma gestão 'managerial'

Cliniques de travail au Chili : trois expériences d'intervention dans une société endommagée par la gestion 'managériale'

Clinics of work in Chile: three experiences of intervention in a society damaged by 'managerial' management

Patricia Guerrero Morales, Manuel Gárate Chateu, Elisa Ansoleaga Imas, J. Marcelo Balboa Gallardo, José Tomas Saffirio Mezzano, Alejandra Nuñez Carbullancautor y M. Valentina Urrutia Fuenzalida

\section{OpenEdition}

Edición electrónica

URL: http://journals.openedition.org/laboreal/15509

DOI: $10.4000 /$ laboreal. 15509

ISSN: 1646-5237

Editor

Universidade do Porto

Referencia electrónica

Patricia Guerrero Morales, Manuel Gárate Chateu, Elisa Ansoleaga Imas, J. Marcelo Balboa Gallardo, José Tomas Saffirio Mezzano, Alejandra Nuñez Carbullancautor y M. Valentina Urrutia Fuenzalida, « Clínicas del trabajo en Chile : tres experiencias de intervención en una sociedad dañada por la gestión 'managerial' », Laboreal [En línea], Volume 15 №2 | 2019, Publicado el 01 diciembre 2019, consultado el 11 abril 2020. URL : http://journals.openedition.org/laboreal/15509 ; DOI : https://doi.org/10.4000/ laboreal.15509

Este documento fue generado automáticamente el 11 abril 2020.

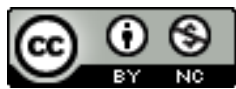

Laboreal está licenciado com uma Licença Creative Commons - Atribuição-NãoComercial 4.0 Internacional. 


\section{Clínicas del trabajo en Chile : tres experiencias de intervención en una sociedad dañada por la gestión 'managerial'}

Clínicas do trabalho no Chile : três experiências de intervenção em uma sociedade danificada por uma gestão 'managerial'

Cliniques de travail au Chili : trois expériences d'intervention dans une société endommagée par la gestion 'managériale'

Clinics of work in Chile: three experiences of intervention in a society damaged

by 'managerial' management

Patricia Guerrero Morales, Manuel Gárate Chateu, Elisa Ansoleaga Imas, J. Marcelo Balboa Gallardo, José Tomas Saffirio Mezzano, Alejandra Nuñez Carbullancautor y M. Valentina Urrutia Fuenzalida

\section{NOTA DEL EDITOR}

Manuscrito recibido en septiembre/2019

Aceptado tras peritaje noviembre/2019

\section{Presentación}

No era depresión, era el capitalismo. Chile Despertó.[1]

1 Esta frase la encontramos en un grafiti en la puerta de una farmacia en el contexto de una de las manifestaciones más grandes en la historia chilena del 2019. Cerca de las instituciones de salud aparecen alusiones al vínculo que existe entre la salud mental, el 
malestar laboral de los ciudadanos chilenos y la organización del trabajo managerial [ [] propias del neoliberalismo instalado a fuerza en nuestro país.

2 Si bien Chile es pionero, el mundo entero ha virado en el mismo sentido en las últimas décadas, impactando la organización del trabajo, con una serie de consecuencias en la subjetividad y en la vida cotidiana. Diversas investigaciones se han concentrado en comprender los alcances de estas transformaciones, tanto a nivel social como subjetivo (Bauman, 2013 ; Boltanski \& Chiapello 1999 ; de Gaulejac, 2005 ; de Gaulejac \& Hanique, 2014 ; Kocyba \& Renault, 2007 ; Rosa, 2010 ; Voswinkel, Gernet \& Renault, 2007). Los autores citados hacen referencia a un "antes" y un "después" a la transformación donde el primer estado tiene cierta nostalgia. Ese "antes" es la industrialización, una socialdemocracia que logró tener pleno empleo, una integración de los trabajadores locales y migrantes (generalmente pertenecientes a las colonias), una organización sindical fuerte. En síntesis, un orden social y una abundancia post guerras mundiales y, en algunos países, momentos de igualdad y de redistribución de las riquezas.

La socialdemocracia con pleno empleo nunca sucedió en Chile, ni con el modelo anterior ni con la llegada del neoliberalismo, por lo tanto, no tenemos una nostalgia de un mundo diferente, de un orden social un poco más justo. La experiencia del gobierno de la Unidad Popular (1970-1973), boicoteado y perseguido, deja un sentimiento dividido, para algunos la tristeza "de lo que no fue" y para otros un alivio "de lo que menos mal no fue". Pese a lo anterior, la caracterización del modelo que proponen los autores extranjeros recién mencionados nos ayuda a pensar la realidad chilena entendiendo que esto viene sucediendo desde hace casi 40 años.

4 Este artículo reflexiona sobre la importancia de pensar una clínica del trabajo que tome en cuenta las características del capitalismo actual y de la organización managerial, presente en la mayoría de las empresas, servicios públicos y privados donde trabaja un 60 \% de la fuerza laboral chilena. Dejamos afuera los otros trabajadores independientes precarizados (llamados "emprendedores") o trabajadores que participan de organizaciones pequeñas, rurales o urbanas, que se caracterizan por artesanado, precarización y bajas remuneración.

5 Este artículo caracterizará este escenario chileno y describirá brevemente tres experiencias de intervención en clínicas del trabajo [3], realizadas en los últimos años en servicios públicos (escuelas, hospitales y servicio público) donde la administración managerial está totalmente instalada. Interesa reflexionar sobre la posibilidad que tienen, en este escenario, las clínicas del trabajo como una forma de intervención que permite a los sujetos pensar como un continuo los procesos individuales, organizacionales y sociales.

6 La gestión en este tipo de instituciones se caracteriza por un modelo, supuestamente "flexible", que aparentemente ofrece más libertad y mejores condiciones laborales. Sin embargo, en la práctica, esta flexibilidad se ha traducido en un exacerbado individualismo y en un mayor nivel de exigencia individual (Bauman, 2013; de Gaulejac, 2011; Honneth, 2006) y menor espacio para el trabajo cooperativo y para aprender de los pares. Los trabajadores se ven enfrentados con nuevas formas de precarización laboral (Offe, 1997 ; Zabala, Guerrero \& Besoain, 2017) que afectan su nivel de seguridad, estabilidad subjetiva (laboral, familiar y personal) y la construcción identitaria que se genera dentro del trabajo (Zabala, Guerrero \& Besoain, 2017). 
7 Autores latinoamericanos y chilenos señalan que la organización del trabajo, en un contexto neoliberal, está fuertemente influenciada, en primer lugar, por una fuerte disminución en el control de la economía por parte del Estado y, en segundo lugar, por el protagonismo y financiamiento de sociedades anónimas donde no existe un solo "patrón", o líder. Esto, en países subdesarrollados como los nuestros, ha significado liberalización y precarización pues se ubican de forma antagónica el fortalecimiento de los factores macroeconómicos y la mejora de las condiciones de trabajo de los empleados (De la Garza Toledo \& Neffa, 2010 ; Ramos 2009 ; Stecher, 2015).

8 Respecto a lo anterior, en el contexto chileno podemos ver una serie de investigaciones que muestran, en distintas áreas productivas, impactos similares en la identidad, subjetividad y salud mental de los trabajadores, todas ellas agudizadas por salarios extremadamente bajos en relación con el costo de vida en el país (Ansoleaga, Dávila, Jara \& Lucero, 2014 ; Sisto, 2012 ; Stecher \& Godoy, 2014 ; entre otros).

\section{Neoliberalismo en Chile : la instalación de la organización "managerial" del trabajo}

9 La aceleración, la competencia y la organización managerial llegaron a Chile antes que a otros lugares del mundo. Nuestro país se ha enfrentado a casi 40 años de neoliberalismo, y mientras se escribe este capítulo nos enfrentamos a una gran protesta social que, por primera vez, lo critica transversal y masivamente, incluyendo demandas de género, educación, pensiones y de la racionalidad instrumental que dañan nuestro ecosistema. La gran movilización social del 2019 propone como forma de lucha, además de la protesta masiva en las calles, salir al espacio público a través de reuniones colectivas en las plazas públicas llamadas "cabildos" en que se discute sobre las condiciones sociales, políticas y personales del país. Estas asambleas tienen como foco conducir el malestar, sistematizarlo y generar propuestas de cambio, donde la más importante es el cambio a la Constitución y la necesidad de una Asamblea Constituyente.

10 En esas reuniones y en las investigaciones (Araujo, 2016), no existe en la población chilena nostalgia por un período anterior : nunca hubo pleno empleo, total desarrollo industrial ni experiencias de colonialismo que dejaran dinero en el país. En los años 60' y 70' algo se empezaba a formar a partir de la nacionalización de las empresas fundadas por los colonizadores del siglo XX, pero ante las demandas de justicia y equidad, estos últimos se coludieron con la oligarquía y los militares para hacer un Golpe de Estado que instaló a la fuerza, no solo un sistema autoritario de gobierno, sino además un modo de vida y trabajo que se constituyó bajo un sistema managerial de organización.

11 La llegada del neoliberalismo comienza a mediados de la década de los 70', junto con el advenimiento de la dictadura cívico-militar liderada por Augusto Pinochet. En este período se pone en marcha un modelo económico de orientación exportadora tras la llegada e influjo de economistas formados en la Escuela de Chicago y toda su batería de organización funcionalista del trabajo defendida por Milton Friedman, conocidos como los "Chicago Boys" (Gárate, 2012). A partir de aquel momento se pone en marcha un nuevo modelo económico que deja su control en manos del mercado, privatizándose gran parte de los servicios e instituciones, dentro de ellas la salud, las pensiones y la educación (dando lugar a escuelas privadas subvencionadas). Sorprendentemente, solo 
permanecieron en manos del Estado aquellas instituciones de salud y seguridad social de las Fuerzas Armadas, a quienes se les protegió de las inseguridades e incertidumbres propias de los regímenes de capitalización individual, situación que se mantiene en la actualidad.

Junto con la entrada masiva de los privados a la economía, las relaciones laborales fueron trastocadas de manera radical. El trabajo, tanto en el sector público como privado, se organizó de un modo managerial y empresarial, sometiendo a los trabajadores a una lógica de competencia con sus pares respecto a la productividad. La fecha simbólica e histórica de inicio es el llamado "Plan Laboral" de 1978, encargado al ministro del trabajo de la época. José Piñera, tuvo como objetivo principal debilitar las organizaciones obreras y sindicales que llegaron a tener gran fuerza durante el gobierno de Salvador Allende. El plan contaba con cuatro ideas principales : (1) el fin de la negociación colectiva por ramas o rubros, (2) la restricción del derecho a huelga con posibilidad de reemplazo de trabajadores, (3) la libertad sindical como forma de generar sindicatos paralelos o bien prohibirlos directamente, y (4) la despolitización de las organizaciones de trabajadores.

13 A lo anterior, debe sumarse el proceso de privatización de las grandes empresas del Estado que habían sido creadas por instituciones estatales desde la década de 1940 (ENDESA de electricidad, Lan Chile aeronáutica, ENTEL telecomunicaciones, etc.). Estas empresas, aparte de emplear a miles de personas, habían constituido toda una cultura del trabajo basada en la acumulación de beneficios, mutualización de riesgos y relaciones laborales horizontales. A ojos de los economistas de la dictadura se trataba de un tipo de empleo ineficiente, de baja productividad, plagado de privilegios y regalías, que fomentaba la politización de los trabajadores. Es así como se impone la nueva idea de la flexibilidad laboral, que, en el papel, aparenta ser muy beneficiosa pero que en la realidad se traduce en mayor facilidad para el despido y precarización de las condiciones del trabajo (horarios, turnos, seguridad, vacaciones, etc.).

Este modelo se terminó de consolidar en los años posteriores a la dictadura, período que coincidió con la Caída del Muro de Berlín y la instalación del llamado Consenso de Washington, el cual promovió la privatización de empresas y las reformas liberales para América Latina. La coalición de partidos de centroizquierda -que gobernaron en Chile desde 1990- no modificó, en lo fundamental, la estructura central del modelo económico heredado de la dictadura (Huneeus, 2016). Es más, sus economistas, también formados fuera de Chile e influenciados por las teorías neoliberales, señalaron, abiertamente que tales reformas y sus modos de gestión eran una condición necesaria para la modernización de los aparatos públicos de todos los países que aspiraban al desarrollo. Chile con un alto nivel de pobreza en los años 70', parecía próspero en los años $90^{\prime}$, vendiéndose incluso como la economía con mayor crecimiento de la región. Esta economía sigue basándose principalmente en la extracción de materias primas y en la exportación de productos primarios, con un constante aumento de sus Tratados de Libre Comercio, tal como ha ocurrido en las tres últimas décadas.

15 La radicalidad de las transformaciones económicas, sumada a la larga duración de la dictadura militar, eliminaron casi por completo las prácticas laborales anteriores a este período. Al menos tres generaciones de chilenos fueron criadas y formadas bajo el paradigma de la productividad capitalista, la cual, si bien ha impulsado índices de crecimiento y acumulación de riquezas, ha generado degradación acelerada del medioambiente y una alta precarización del empleo que no la justifican. 
16 En términos sociales, entre 1973 y 1990 se prohíbe constitucionalmente agruparse, formar organizaciones sindicales y partidos políticos. Solo la iglesia podía seguir funcionando y un sector progresista de ella albergó movimientos sociales. Los chilenos temen al colectivo y desconfían de estar en grupo, cerrándose incluso carreras universitarias de las Ciencias Sociales. Por muchos años, los trabajadores valoraban "ir de la casa al trabajo, del trabajo a la casa", sin espacio para la militancia, el ocio o estar con otros que no fueran del núcleo más cercano o familiar.

Diferentes estudios (PNUD, 2017) demuestran que, hoy en día, los chilenos trabajan muchas horas, producen relativamente poco, tienen salarios bajos, pasan largas horas en el transporte público y sufren distintas enfermedades asociadas a estas condiciones. Lo anterior demuestra que la aparente bonanza, de los últimos 30 años, se sustenta en una precarización de la vida y en un consumo basado en un alto endeudamiento. La imagen de un país con "buena salud económica" ha sido solamente una fachada que refleja, únicamente, el lado positivo, el del crecimiento económico. Esta situación puede ser cuestionada pues, a la fecha, Chile continúa siendo un país sin un desarrollo productivo real y que basa su economía en la exportación de commodities. Además, y justamente por la privatización de sus servicios, en detrimento de las estructuras públicas, convive con una tasa de inequidad interna mayor que la de sus países vecinos.

Varias organizaciones han entrado en crisis por no dar abasto frente a las demandas de una población que hace público su malestar. Las pensiones con ahorro individual muestran que los trabajadores obtienen menos de un tercio de su salario, la educación chilena tiene malos resultados pese a la inversión de recursos y es una de las más desiguales de América Latina. Además del malestar social, sabemos que hay problemas en el trabajo, porque existe un consumo masivo de medicamentos psiquiátricos y un aumento sistematizado de ausentismo laboral.

19 La respuesta, por parte de los diferentes gobiernos inspirados en políticas públicas con estándares internacionales, ha sido la promulgación de nuevas políticas públicas en salud mental ocupacional. Entre estas está la Ley de Riesgos Psicosociales del trabajo que exige a los empleadores aplicar evaluaciones de riesgo a sus trabajadores y luego entregar medidas de mitigación para reducirlos. Esta legislación está siendo una oportunidad para consolidar un trabajo de escucha colectiva y no individualizada (Zabala, Guerrero \& Besoain, 2017).

20 En este contexto, las clínicas del trabajo aparecen como línea de intervención que permite pensar la salud mental tomando en cuenta los factores individuales, sociales y políticos. Nuestra apuesta no es solo hacernos cargo de la creciente demanda por abordar y mitigar el malestar dentro del trabajo, producido por estas nuevas formas de organización managerial, sino pensarlas psicosocialmente para que los actores puedan realizar acciones, que permitan cambios reales, distinguiendo aquellas que están a su alcance de aquellas que necesitan acciones organizacionales o políticas.

\section{Las clínicas del trabajo en Chile : aspectos comunes para resistir}

21 Dentro de la aproximación de las "clínicas del trabajo", para el caso chileno, se logran aliar aquellos investigadores que trabajan desde el socioanálisis, el análisis institucional, la psicodinámica del trabajo, la sociología clínica y otras corrientes 
psicosociales del trabajo. Luego de algunos años de preguntarse por lo común que tienen estas teorías e intervenciones clínicas logramos acuerdo en los siguientes puntos (Zabala, Guerrero \& Besoain, 2017).

En primer lugar, las clínicas del trabajo piensan la experiencia laboral a partir de los límites y conflictos que impone el trabajo. A diario ocurren imprevistos que demandan nuevos saberes y habilidades enfrentando al sujeto con lo real del trabajo. Aquí, el fracaso aparece como una posibilidad inminente, como una experiencia afectiva displacentera que moviliza a resolver la situación a través del análisis y el pensamiento. (Dejours, 2009 ; Zabala, Guerrero \& Besoain, 2017). El conflicto y la dificultad son el motor del trabajo que ayuda a mejorar la capacidad creativa de los sujetos.

En segundo lugar, se parte de la experiencia subjetiva y de la implicación para pensar las problemáticas asociadas al trabajo (Lhuilier, 2006; Zabala, Guerrero \& Besoain, 2017). A través de la utilización del propio cuerpo e identificando las posibilidades de creación y transformación, el trabajador puede comprender y encontrarse ya no solo con el malestar dentro del trabajo, sino también con el placer que este le proporciona (Zabala, Guerrero \& Besoain, 2017). El sujeto no es un recurso en el trabajo, sino que es un lugar donde se construye encarnado y es desde esa posición que aporta a la sociedad.

En tercer lugar, el sujeto está siempre con otros y/o en relación con otros aún cuando su actividad pueda parecer solitaria. Esta relación real o simbólica porque el trabajador siempre está expuesto al reconocimiento del trabajo de sus pares, de sus usuarios y de sus jefes (Barus-Michel, 2004; Dejours, 2009; Lhuilier, 2006; Zabala, Guerrero \& Besoain, 2017). Lo relevante de tal encuentro es que afecta, inevitablemente, el resultado de la tarea, según favorezca o dificulte el trabajo cooperativo (Clot \&Lhulier, 2010 ; Dejours, 2009 ; de Gaulejac, Giust-Desprairies \& Massa, 2013). Esta idea cuestiona el concepto de "carrera individual", tan propio de la ideología neoliberal. Las clínicas del trabajo, incorporan nociones como la de imaginario colectivo, reconocimiento, cooperación, grupalidad e institución (Zabala, Guerrero \& Besoain, 2017).

En cuarto lugar, todas las clínicas mencionadas analizan las maneras en que los sujetos construyen "experticia" en el trabajo. Nos damos cuenta de que los trabajadores sienten un impulso por mejorar su actividad laboral y que se esfuerzan para mantener un buen desempeño. Desde esta perspectiva, las problemáticas del trabajo se analizan tomando en consideración elementos individuales y colectivos entendiendo que ambos aspectos son necesarios para que el sujeto acceda a la transformación de su espacio (Lhuilier, 2006 ; Zabala, Guerrero \& Besoain, 2017). Aquí las investigaciones convergen con aquellas orientadas a las historias de la profesión, las identidades profesionales y los manuales de oficio que nos permiten darnos cuenta que los trabajadores se implican en su labor (Clot \& Lhuiller, 2010 ; Guerrero, 2008 ; Lhullier, 2006 ; Zabala, Guerrero \& Besoain, 2017).

Por último, al momento de intervenir, estas clínicas apuntan a generar procesos reflexivos colectivos. Para lo anterior se ofrecen a la organización todas sus herramientas teóricas, siempre acompañadas de un trabajo de elaboración entre el interventor y los participantes. Se espera que los trabajadores, en conjunto, puedan luego construir y coordinar su propia acción (Zabala, Guerrero \& Besoain, 2017).

Las clínicas del trabajo chilenas no buscan entregar prescripciones a las organizaciones ni adaptar los sujetos a los nuevos escenarios del mundo laboral. El foco consiste en 
comprender y describir las dificultades humanas en el trabajo, cuestionando sus transformaciones y atendiendo fenomenológicamente las dificultades psicosociales de la organización del trabajo (Zabala, Guerrero \& Besoain, 2017).

Las características de las clínicas del trabajo son un verdadero desafío en Chile considerando que durante 17 años de dictadura el trabajo grupal, las investigaciones cualitativas, las agrupaciones sindicales y las carreras de las Ciencias Humanas fueron perseguidos. Así, la generación que tiene más de 40 años, fueron educados en dictadura, sin experiencia de aprendizaje cooperativo en lo formal, donde la experiencia grupal está asociada a la persecución y el miedo.

Volver a estar en grupo y tener placer de crear con otros, es una tarea desconocida y con la que las nuevas generaciones tampoco están familiarizadas porque la educación también es una carrera individual. Por lo tanto, son varias generaciones las que no saben cómo habitar lo colectivo de manera sana y creativa. Como se señaló, el 2019, comienzan a armarse "cabildos" en que se empieza a crear pensamiento colectivo, antes de eso, lo colectivo carecía de legitimidad.

\section{Tres experiencias en clínicas del trabajo}

Hasta ahora se han presentado las principales características de la situación laboral en Chile y aquello que reúne a las clínicas del trabajo, construidas para hacer frente a este complejo y paradojal escenario. Por lo mismo, es pertinente mostrar tres experiencias concretas de aplicación de estas clínicas. La idea es reflejar el alcance de sus teorías e intervenciones dentro de organizaciones estatales y privadas con gestión managerial. Cada una de las experiencias permite ejemplificar intervenciones desde diferentes clínicas del trabajo : clínica educacional, clínica psicosocial del trabajo y socioanálisis.

La primera experiencia ocurre en un contexto de formación continua (capacitación) para profesores de escuelas y liceos públicos (formación de docentes en ejercicio). La segunda en un servicio de salud y la tercera en un servicio público. Estas son tres experiencias diferentes en que las clínicas del trabajo están buscando su lugar.

Muchos otros autores han quedado fuera de este artículo, pero elegimos aquellos que permitieran reflejar la diversidad de formas en que se ha intentado dar lugar al sufrimiento humano en el trabajo y las formas de elaborarlo desde y con el colectivo.

\subsection{Clínica Educacional : una propuesta aplicada a la Formación de Docentes}

33 Tal como se plantea en la introducción, la sociedad chilena y en particular la educación está fuertemente influenciada por lógicas empresariales (Guerrero, 2017 ; de Gaulejac \& Guerrero, 2017). Por lo mismo, para entender esta intervención clínica es necesario conocer algunos antecedentes particulares. Lo primero es que las escuelas no tienen financiamiento basal, sino que se rigen por un sistema de "voucher" donde el dinero que reciben depende de la cantidad de alumnos y alumnas que logran reclutar con sus resultados. Por lo mismo, las escuelas tienen un sistema de rendición de cuentas de altas consecuencias en relación con su calidad, medida principalmente con los resultados de prueba estandarizada. Además, existe una "agencia de la calidad" para analizar estos resultados y clasificar a las escuelas en diferentes categorías de 
desempeño e informar de ello a las familias que eligen escuelas. Se busca, tal como en el sueño de Milton Friedman, que los padres "voten con los pies" y que consecuentemente se cierren las escuelas con mal desempeño.

Con este tipo de organización, la educación chilena es una de las más segregadas y desiguales del mundo (OECD, 2015, UNESCO, 2017), ya que los alumnos con buenos rendimientos asisten a escuelas bien clasificadas y los alumnos con bajos rendimientos llegan a las escuelas con bajos resultados. Esta realidad es muy difícil de transformar, porque la lógica neoliberal está instalada y validada en Chile. Muchos padres creen que tras conocer el rendimiento de las escuelas podrán elegir con mayor libertad. Frente a esta realidad ningún gobierno democrático ha decidido realizar transformaciones profundas, posiblemente porque puede generar mucha oposición por parte de las familias que sienten en esa libertad un beneficio. El Neoliberalismo está instalado en la población chilena y espera de la educación, pública o privada, un servicio de calidad.

Paliar las diferencias sociales, se han implementado progresivamente, entre el 2000 y 2017, una serie de reformas orientadas a garantizar la igualdad de derecho a la educación (MINEDUC, $2015\left[{ }^{4}\right]$ ). En 2015 se promulga la "Ley de Inclusión" que genera un nuevo avance en este proceso de democratización educacional, porque pone desafíos curriculares y metodológicos a los profesores. Pero además pone fin a la posibilidad de lucrar con la educación estatal, impidiendo la selección de los estudiantes a las escuelas - se asignan de manera aleatorias - y entregando recursos para que los profesores tengan ayuda en la atención de los niños con diversas dificultades (MINEDUC, 2015/2017) [5].

En términos de oficio, esta ley agrega prescripciones a la labor docente. No sólo deben obtener buenos resultados en las pruebas estandarizadas, sino que además deben aprender a trabajar con otros profesionales y cambiar su oficio en la sala de clases, orientándola hacia un aula diversa que promueva el aprendizaje colaborativo. Lo anterior demanda a los docentes estudio y mejoramiento permanente, además de la necesidad de re-aprender a valorar las diferencias entre los alumnos y manejar las diferentes condiciones de aprendizaje.

Es la Facultad de Educación de la Pontificia Universidad Católica de Chile que ha dado la posibilidad de insertarse en procesos de formación continua docente (formación de profesores en ejercicio) en diversos temas relacionados con las problemáticas de las desigualdades en las escuelas (violencia escolar, trabajo con la migración, trabajo con estrategias digitales, entre otros). A partir de los cursos impartidos ha sido posible generar un diálogo y levantamiento, junto con los profesores, sobre el impacto subjetivo que les significa la implementación de estas transformaciones disciplinares. Aproximarse a este fenómeno, desde una clínica educacional, permite contar con los instrumentos conceptuales y metodológicos, necesarios para dar cuenta sobre la movilización subjetiva que esto les significa, tanto a nivel individual como colectivo.

En este contexto aparece la tarea de enseñar "Estrategias diversificadas de educación y aprendizaje cooperativo" desde las clínicas del trabajo. Es decir, enseñar nuevas metodologías de enseñanza-aprendizaje que se centren en la sala de clases y que pongan foco en la cooperación de los estudiantes.

La formación se lleva a cabo a partir de un trabajo situado que apunte al contexto de cada escuela y que considere central la generación de un diálogo entre los saberes disciplinares, para visibilizar y valorar el manejo de los docentes frente a la normativa, 
porque este también implica un saber disciplinar. El dispositivo formativo debe incorporar el saber de oficio de los profesores como parte de un encuentro de saberes, necesario para una mejor asimilación de las tecnologías propuestas por la normativa. Esta formación es desde la clínica del trabajo en educación.

Se parte del principio que toda formación debe realizarse de manera implicada. Entonces, los contenidos que se enseñan a los profesores son en base a su experiencia laboral y de vida. No existe ningún proceso de formación que no esté encarnado en la biografía, en los sentimientos, situado en la organización que ocurre y en el contexto del país. En este sentido, los dispositivos metodológicos tomados en especial de la sociología clínica se adaptan a cada realidad. Por ejemplo, en una sesión de Organidrama (Gastal de Castro \& Guerrero, 2013) en que se teatraliza un conflicto, la palabra "conflicto" no puede ser explicitada sin previamente dar una definición, un contexto y un espacio para pensarlo. Es necesario considerar que la sociedad chilena tiene poca experiencia en discutir en grupos y tiene un trauma reciente producto de la dictadura y luego de la política de los consensos, por lo que la asociación entre la palabra conflicto, resolución violenta, temor, emerge de manera inmediata.

El segundo elemento que hace esta intervención clínica es que trabaja desde la experiencia subjetiva de los trabajadores y confía en la transformación de su lugar de trabajo para hacerse experto en su oficio, siendo el trabajo un lugar que puede dar satisfacción. Las influencias del marxismo tradicional en el pensamiento crítico han instalado en el imaginario la idea de que es necesario cambiar primero las estructuras sociales y luego pensar en hacer cualquier cambio individual. Pese a coincidir con el análisis que hacen sobre las injusticias y necesidades de cambio en nuestro país, la clínica educacional sigue un camino diferente. Se toma de la sociología clínica y de la psicosociología la idea de que existe una articulación, una relación indisociable, entre los procesos sociales y los procesos individuales. En ese sentido, se comprende que para que una sociedad logre cambiar se necesita un proceso individual además de las transformaciones sociales. En este sentido, la frase de Vincent de Gaulejac "el individuo es producto de una historia de la que trata de transformarse en sujeto" (de Gaulejac, 2000, p. 55) inspira este trabajo porque muestra la posibilidad que tiene el sujeto de ser actor de su vida y constructor de su propia historia.

Para los interventores, frente al desamparo de la educación de mercado, tal articulación aparece como una herramienta para trabajar con los equipos de docentes, en especial porque los profesores no están logrando los desempeños esperados por la escuela. Por lo mismo, la intervención considera la revisión de las historias personales y la de la organización, conocer las aproximaciones en el ámbito de la educación e invita a la construcción de micro experiencias, contextualizadas, que apunten al cambio. Esta idea se complementa, además, con las nociones de agencia (Priestley, Biesta \& Robinson, 2015) que, desde algunas teorías críticas, nos muestran la interrelación de los procesos sociales y los procesos individuales. Un desafío que se tiene pendiente es poder "politizar" el sufrimiento, mediante los procesos de defensa de los trabajadores como ya lo realizan equipos en Canadá de abogacía y mediación (Viviers, Boulet \& Hamelin, 2018).

El dispositivo de formación en "Diversificación de la enseñanza y aprendizaje cooperativo" se concentra, en un inicio, en el despliegue de un conjunto de conceptos y tareas pre-formativas y performativas dentro del grupo docente. 

potencia a partir de la de-construcción de la implicación frente a la inclusión escolar permitiéndole a los docentes poder dimensionar lo que en la teoría se busca : validar la diversidad y reconocer en heterogeneidad un soporte y un potenciador del aprendizaje. desde un foco en la particularidad de los niños y niñas "difíciles" (a los que hay que adecuar y hacer más simples las tareas) hacia el reconocimiento y focalización en el 
colectivo, permitiéndoles visualizar el conjunto de estudiantes, sus necesidades y potencialidades, y enriqueciendo el aprendizaje grupal y cooperativo.

Respecto de la noción de agencia. Este trabajo permite la real comprensión de la agencia del profesorado, no se siente un actor pasivo que quiere que cambie el sistema neoliberal, ni un actor ciego a las ataduras de este mismo sistema. La idea es ver qué concreto puede cambiar en su práctica que lo conecte con el sentido de lo que hace y con la crítica a la organización empresarial en educación. Los principales impedimentos para hacer bien su trabajo, no es su voluntad, sino el sistema de evaluación empresarial con pruebas estandarizadas.

En este sentido, este curso de formación diseñado desde la clínica del trabajo posibilita una aproximación privilegiada al fenómeno educacional, permiten la apertura y flexibilidad para articular dispositivos a través de la vivencia y la reflexión grupal frente a situaciones cotidianas entendidas en su complejidad social, y que operan en favor de la emergencia de los recursos vivenciales y técnicos del grupo docente. Además de someterlos a un análisis crítico y situado sobre su contexto y tarea.

\subsection{Clínica psicosocial del trabajo : una propuesta de intervención}

Como se señala en la introducción, una de las consecuencias de las transformaciones en el mundo del trabajo, y que afecta en sus distintas áreas, es la creciente necesidad de las organizaciones de contar con intervenciones pertinentes y complejas para abordar el estrés y el sufrimiento derivado del trabajo. Las empresas e instituciones públicas están expuestas a grandes demandas de evaluación y se necesita una mayor atención de las personas y las organizaciones. Las empresas cuentan con soluciones ligadas al "autocuidado" como estrategias de relajación y felicidad en el trabajo, pero que no cuestionan la organización social del trabajo y las condiciones laborales propias de las exigencias manageriales y neoliberales.

Varias universidades han comenzado a estudiar el mundo del trabajo, incluso hay grandes fondos estatales que se asignan a esta tarea [ $\left.{ }^{6}\right]$ Sin embargo, la intervención o la investigación-intervención tienen un lugar menor. Frente a este análisis, la Clínica Psicosocial del Trabajo (CPT) fue elaborada por el Área de Salud Mental y Trabajo, dentro de la Clínica Psicológica de la Universidad Diego Portales en 2013. Esta propuesta considera dos modelos y/o perspectivas útiles para comprender el abordaje del sufrimiento en el trabajo : la sociología clínica o psicosociología (de Gaulejac, 2000) y la psicodinámica del trabajo (Dejours, 2009).

57 Como se trata de una experiencia en clínica del trabajo, las intervenciones desde la CPT se desarrollan en forma grupal con participación voluntaria y asistencia libre. Esta estructura busca dar cabida a la aparición de temas emergentes dentro del medio laboral, y permite generar una participación responsable y autónoma. Para guiar las sesiones se sugiere la participación de, al menos, dos profesionales y, en lo posible, contar con el apoyo de una tercera persona cuyo rol sea el registro en audio y escrito de la sesión. Para garantizar la continuidad del trabajo en las reuniones se utiliza como instrumento principal una Bitácora, la cual consigna la historia de una sesión a la siguiente, los temas centrales que se abordaron, acuerdos, conclusiones, propuestas, etc.

58 Esta bitácora es realizada por los profesionales de grupo y tiene como función dar continuidad a los procesos, para eso se lee al inicio de cada sesión y se completa y/o 
modifica con la opinión de todos los asistentes. El dispositivo entiende que todo lo que pasa en la relación es parte de la intervención, desde escuchar la demanda hasta ampliar posibilidades y participantes, establecer límites y acceder a conversaciones con diferentes actores. Esta metodología tiene como principal tarea dar un espacio a la problemática de las instituciones y al sufrimiento individual, pero además crear soluciones en conjunto que transformen la organización del trabajo.

Luego de una serie de intervenciones, esta clínica psicosocial del trabajo instala una metodología, que tiene pasos detallados que además se adecúan a las distintas realidades y terrenos.

El dispositivo comienza por recoger una queja institucional, la que puede ser planteada por directivos, jefes, o por el malestar de sus funcionarios a través de algún dirigente de los trabajadores. El cómo se comunica la petición ilumina el problema, pues en su 'forma' están presentes características propias de la organización. Para quien acoge la demanda, una primera tarea consiste en comprender cómo ésta surge la, por qué se requiere su abordaje, por qué es 'tramitada' de este modo y de qué manera esto representa la forma que la propia organización tiene de entender y enfrentar situaciones problemáticas (efecto hologramático, sentido e historia institucional). Una vez resuelta esta primera tarea, la cuestión siguiente es resolver cuál es la expectativa de quien la solicita y los aspectos formales de funcionamiento del trabajo.

61 Luego de este trabajo de "queja institucional", desde la lógica de la demanda y la explicitación de los temas a trabajar, es necesario preguntarse quiénes deben participar y a qué unidades pertenecen. Así, se negocian no solo los aspectos formales sino también los límites de participación, en lo posible en función de la unidad a la que pertenecen. Mientras más interdependiente es el grupo será más fácil buscar alternativas y posibilidades de acción. Una vez acordados los participantes, se explicitan y acuerdan las condiciones que la institución ofrece para que las personas puedan asistir y se procede a hacer contratos colectivos.

62 La etapa siguiente corresponde a la intervención grupal dentro del sistema laboral que no se crea con esta idea, pero que tienen efectos psicoterapéuticos. La tarea es generar un espacio de confianza, de validación de las distintas versiones sobre las dificultades laborales y los sufrimientos personales. De esta forma es posible colectivizar el sufrimiento : si hay personas que dentro del medio laboral están en malas condiciones emocionales, relacionales o psicológicas, no son ellas las responsables. Esta es una idea opuesta a lo que se concibe dentro de un sistema neoliberal y en una organización de trabajo managerial, donde las responsabilidades del sufrimiento humano son individuales. Aquí la sistematización de esta información en Bitácoras no solo da continuidad del trabajo sesión a sesión, elabora y pone en palabras, exterioriza el sufrimiento de las personas, pero también las causas sociales y las consecuencias para la organización y para ellos mismos.

63 Concretamente, cada una de las sesiones duran entre una hora y media y dos horas, donde el tiempo se reparte en tres momentos claves : uno de inicio, uno de desarrollo y otro de cierre. El momento de inicio da un tiempo acotado a la lectura de la Bitácora de la sesión anterior y se abre a comentarios y discusión con los participantes. El momento de desarrollo es el que debe ocupar el mayor porcentaje de tiempo y está destinado a comentar el/los temas expuestos para la sesión, modelando formas de desacuerdo, evidenciando consecuencias y desafíos, y dando la posibilidad a todos los participantes de expresar su opinión. Es relevante para los profesionales guías recordar que no se 
busca el consenso, que la divergencia es lo que se espera para poder construir nuevas alternativas. El momento de cierre debe ser intencional, dejando al menos 15 minutos para conocer el estado en que están los participantes y cómo se van, así como también para fijar el tema principal de esa sesión.

Además, existen tres tipos de sesiones diseñadas para abordar diferentes momentos y objetivos del proceso. Se les caracteriza por su temporalidad, es decir, se llaman de inicio, de desarrollo y de cierre. En las sesiones de inicio se establece un espacio de confianza con normas compartidas de funcionamiento, es especial la confidencialidad, respeto, participación y compromiso. La meta principal es acordar el qué y el cómo se trabajará. Los profesionales deben estar atentos y abiertos a las propuestas del grupo (imprevisibilidad), independiente de los planteamientos iniciales de quien realiza el contacto o la petición.

En las sesiones de desarrollo es el grupo el que fija los objetivos a trabajar, por lo tanto, dependerá de cada grupo, de sus necesidades y de los temas sus características. En función de la tarea grupal es el número de sesiones que se les proponen, la duración, el lugar, etc.

El cierre será acordado considerando lo establecido en las sesiones de inicio con los mismos participantes. El grupo puede dar continuidad al trabajo armando un grupo autónomo (sólo entre profesionales) o continuar con las sesiones. Al finalizar se entrega un informe escrito que rescata las principales conclusiones, cambios o sugerencias para entregar a quienes gestionaron la petición original durante el primer contacto. Este documento es consensuado y revisado por las/os integrantes del grupo, previo a su envío y tiene como foco la comunicación de las dificultades encontradas en la organización del trabajo.

Una vez entregados los documentos se acuerda con el grupo una manera de seguimiento de las acciones interpretadas. El equipo interventor no solo se compromete a visibilizar el sufrimiento, sino también a hacer un seguimiento con la información recopilada.

Este modelo ya ha sido utilizado con trabajadores del sector público (trabajadores de salud, justicia y de sitios de memoria y Derechos Humanos demostrando, en terreno, que permite trabajar con el malestar del trabajo. Por lo mismo vale la pena compartir una experiencia real de aplicación de esta estrategia.

La intervención seleccionada es una que se realizó con un grupo de trabajadoras de la unidad de oncología pediátrica dentro de un hospital público de alta complejidad. En esa ocasión se trabajó en la elaboración del dispositivo 'Construyendo espacios de colaboración : la importancia de lo colectivo en la organización del trabajo' el que refleja la necesidad de ofrecer alternativas de abordaje en relación a los efectos que el trabajo tiene en la salud de las y los trabajadores.

Durante un período de aproximadamente 2 meses se realizaron 9 sesiones en las que participaron 8 mujeres de distintos estamentos de la unidad: Enfermeras, Secretarias, Técnicos Paramédicos, Auxiliares, Médicos y Psicólogas. Respecto al número de asistentes por sesión hubo un promedio de 4 personas, con un máximo de 6 .

71 Para caracterizar la organización es necesario decir que la Unidad de Oncología considera un área de trabajo Ambulatorio y otra de Hospitalizados, las cuales presentan algunas diferencias entre sí. Por ejemplo, en el área Ambulatorio hay una alta demanda de niños cuyo tratamiento varía en duración y el trabajo se realiza exclusivamente en 
horario diurno (8 a 17 horas). Por otro lado, en Hospitalizados, además de camas para internación, se encuentran las salas de cuidados paliativos donde es posible que fallezcan niños. En lo organizativo cuentan con distintos tipos de jornada laboral y en esta sección es posible encontrar diversos sistemas de turnos. Ambas secciones comparten el objetivo principal de cuidar a niños que han sido diagnosticados con cáncer. Lo anterior implica entregar el tratamiento correspondiente en las distintas fases de la enfermedad e informar oportunamente a los familiares o cuidadores de los pacientes sobre su estado de salud. Es así como este servicio se caracteriza por su complejidad y no sólo a nivel de competencias clínicas, sino también relacionales, puesto que en él se viven, cotidianamente, situaciones críticas que se acompañan de altos montos de estrés y desgaste emocional. Estas situaciones implican la contención simultánea, tanto de las familiares como de los propios funcionarios, frente a la gravedad de la sintomatología y al eventual fallecimiento de las/os niñas/os. despliegan para afrontar el sufrimiento tienen que ver con la fantasía de la renuncia a la institución, la negación de las circunstancias de malestar (no compartir lo que sienten, pues puede ser "mal visto"), la dificultad para distinguir espacios laborales de los no laborales y la inexistencia de "auto-autorización" para hacer una pausa cuando están afectadas. También emerge como necesidad, para un mejor enfrentamiento de la tarea, la posibilidad de involucrar a más personas que participen en la clínica Psicosocial del Trabajo, lo que podría contribuir a generar cambios estructurales en la organización del trabajo siendo fundamental para ello coordinarse para mantener el servicio operativo. 


\subsection{Socioanálisis : el análisis del rol en la organización.}

78 La experiencia chilena en Socioanálisis tiene su origen en el Departamento de Administración de la Facultad de Economía y Negocios de la Universidad de Chile, donde un grupo de profesores ha realizado diferentes procesos de investigación acción, sumado a programas de formación como Diplomados y Magíster en Gestión de Personas y Dinámica, todos desde la perspectiva socioanalítica. El grupo que desarrolló la intervención de este artículo colabora con este equipo de profesores en temas particulares, y a la vez forman parte de la Consultora Sintoniza, dedicada a la gestión de personas. Además, contribuye a generar cambios en las organizaciones, desarrollando espacios de reflexión - acción en sus procesos de consultoría y formación implementados. En términos generales, las iniciativas utilizadas vienen desde el enfoque socioanalítico.

La tradición Socioanalítica incorpora el pensamiento psicoanalítico, la teoría de sistemas y el estudio de las relaciones de grupo. Esta tradición emerge a partir de un trabajo seminal realizado durante la segunda mitad del siglo XX en el Instituto Tavistock en Reino Unido. Desde esta orientación se ha contribuido a la exploración de dinámicas que enfrentan las organizaciones contemporáneas y en el diseño de metodologías de intervención, ayudando a abordar procesos de cambio que permitan llegar a los ambientes organizacionales actuales.

Esta corriente indaga en aspectos inconscientes de la realidad, poniendo al descubierto tabúes de la vida de las organizaciones y de la sociedad. Por ello, se considera una metodología adecuada para aproximarse a las experiencias subjetivas. Una tarea es que se toma una postura de sospecha frente a la realidad convencional, reconociendo que esta abarca aspectos ocultos y subterráneos, y que además son complejos de llevar a la superficie pues ese proceso implica contactarse con tensiones, conflictos y ansiedades de difícil discernimiento y solución. El socioanálisis comprende, también, que los hombres y mujeres que participan de una institución suelen estar sometidos a grandes presiones, teñidas por definiciones de autoridades e instituciones, hechos y situaciones, pensamiento positivista, saber de expertos, etc.; de esta manera reconoce que existe una realidad en la sombra, donde quedan ocultas dimensiones trascendentes que, de ser conocidas, pueden traer entendimiento y liberación (Acuña \& Sanfuentes, 2013).

81 Precisamente, el socioanálisis busca liberar las experiencias subjetivas de los trabajadores ubicando este punto como foco de aprendizaje. Además, destaca el trabajo conjunto, porque reconoce que el conocimiento se genera de manera colectiva mediante la interacción entre el cliente y consultor. Aprendizaje que estimula el pensamiento crítico, develando mitos y destrabando el desarrollo de las organizaciones. Imprescindible para que se generen acciones de cambio (Acuña \& Sanfuentes, 2013).

El socioanálisis cuenta con diversas metodologías que permiten abordar el trabajo en el espacio organizacional de manera más eficiente, considerando el contexto, el sistema y las personas en roles. Una de estas metodologías es el Análisis del Rol Organizacional (ARO), la misma que fue utilizada en el caso que dará a conocer en esta presentación.

Primero, es importante señalar que ARO es un método de coaching y consultoría que busca proveer a los clientes un espacio de reflexión y aprendizajes de sus propias experiencias en el trabajo. De esta manera, se genera un espacio de colaboración para que los grupos y personas en rol, en forma autónoma y responsable, encuentren 
solución frente a los dilemas o dificultades que experimentan en el ejercicio de sus roles organizacionales (Acuña \& Sanfuentes, 2013).

En el ARO se entiende el Rol como la intersección entre la persona y el sistema, principio regulador que se sostienen en la mente y modela la forma en que se emiten los juicios y las acciones cuando se está en rol. La autoridad, para ejercer un "rol", viene desde adentro (sí mismo) y desde afuera (sistema), por lo mismo estar en rol es una capacidad y una persona tiene tantos roles como sistemas en los que participa (Acuña \& Sanfuentes 2008). Es importante mencionar también que lo que se observa no es un rol, sólo las conductas.

El Rol puede ser entendido y analizado desde dos dimensiones: la dimensión organizacional y personal. En la dimensión organizacional es posible explorar las expectativas que tiene la organización sobre cómo se deben llevar a cabo las tareas. Estas expectativas pueden estar mediadas por la cultura de la organización, su historia y su situación actual. Luego, en la dimensión personal del Rol es posible entender las expectativas de la persona que ejecuta las tareas, sus necesidades y las habilidades que posee. El rol, por lo tanto, abarca un área de convergencia entre el sistema y la persona, tal como muestra el esquema a continuación (Acuña \& Sanfuentes, 2008).

Cuando una persona logra alinear estas dos dimensiones se incrementa el desempeño, en términos de la calidad del trabajo como también en las emociones con las que lo vincula. La persona es más productiva y tiene una actitud positiva. Al tener mayor claridad del Rol es posible que la persona pueda transformar el Rol que tiene en la mente y alinearlo con la idea que tiene el sistema respecto de este. La finalidad central del ARO es colaborar con las personas para que puedan administrarse a sí mismos en su Rol. Que pueda hacer uso de la autoridad personal, de forma autónoma y responsable, rompiendo así el mito de que únicamente la élite tiene la capacidad de transformar su Rol (Acuña y Sanfuentes, 2008).

La intervención socio analítica que se presenta fue realizada en un ministerio del Gobierno de Chile, donde sus autoridades contactan al equipo consultor para solicitar apoyo. El motivo es el aumento de salidas de funcionarios producto de la ausencia de posibilidades de desarrollo dentro de la institución. La tarea asignada fue contribuir al desarrollo laboral de sus funcionarios existiendo, por ende, la necesidad de pensar su estructura. El programa tuvo como objetivo entregar las herramientas para desarrollar las competencias necesarias que sirvieran para responder tanto a los requerimientos de sus cargos como a sus propias expectativas de desarrollo, e indirectamente generar así un mecanismo para la retención de talentos. Se diseñó el programa "Gestión de Desarrollo Laboral".

En este contexto, el Programa tenía como objetivo proveer un espacio de reflexión a los funcionarios participantes, permitiéndoles explorar las dimensiones de su Rol y sobre ello analizar su vocación y el sentido de su trabajo. Este objetivo se logró a través de la instalación de un dispositivo de trabajo en torno a Plenarios, Grupos Pequeños y Grupos de Aplicación, mediante los cuales se facilitaban los procesos reflexivos y las tareas de cada grupo. Específicamente se buscó generar en los participantes un proceso de toma de conciencia respecto al Rol que ejercían en su trabajo, entregando espacio para generar posibles acciones de cambio o innovaciones orientadas a una mayor conexión entre sus expectativas y las de la institución. La hipótesis sobre la que se trabajó, a la hora de diseñar el programa, fue que, si el funcionario hallaba algún grado de 
coherencia, entre la dimensión personal y organizacional del rol, esto impactaría positivamente en el grado de sentido hallado en el trabajo.

El programa de "Gestión de Desarrollo Laboral" contempló 20 horas de trabajo presencial, además de 16 horas de ejercicios de aplicación monitoreadas por el equipo académico a través de una plataforma virtual. El grupo estuvo conformado por 120 personas y se dividieron en subgrupos de 30 personas. En la plataforma señalada se podía acceder a bibliografía de apoyo, contactarse con los demás participantes y entregar ejercicios de aplicación para que así los asistentes, pudieran darle continuidad a su aprendizaje.

El grupo de trabajo funcionó como una organización temporal de aprendizaje, donde se experimentaron las dinámicas que surgían en el "aquí y ahora" y donde cada participante vivenciaba la interacción de los distintos roles, convirtiéndose en un "observador participante" de la dinámica y evolución del grupo. La experiencia de trabajo con el grupo permitió extrapolar el aprendizaje a las realidades cotidianas de cada participante (Acuña \& Sanfuentes, 2013).

Resultados:

Del análisis de las evaluaciones y comentarios de los participantes sumado a la experiencia de los consultores podemos señalar los siguientes hallazgos

Respecto la percepción de las posibilidades de desarrollo laboral en instituciones públicas. Los participantes de los talleres una vez que tomaron conciencia de su rol laboral lograron ampliar su horizonte de posibilidades, concluyendo que existe desarrollo laboral más allá de la estructura jerárquica. Así, consiguieron ver a la institución de una forma transversal, de forma subjetiva (historia, memoria de la institución, fantasía consciente o inconsciente del rol) lo cual, tal como mencionamos más arriba, fortalece el liderazgo y la autoridad personal permitiendo el desarrollo del pensamiento crítico y una mayor autonomía en la toma de decisiones respecto al desarrollo laboral.

Respecto al impacto de la reflexión en el cambio social. El programa permitió a los participantes "reflexionar" respecto de sus roles y conectar o reconectar con su vocación, a través de la identificación de las ideas y sentimiento con los cuales vinculaba y "vivía" su trabajo; en este punto fue posible que cada participante construyera el sentido de su trabajo, sentido que es particular y determinado por las expectativas y deseos de cada persona. Este resultado puede ser entendido como una evidencia de la capacidad de los sujetos para asumir nuevas posturas respecto de su relación con el trabajo y la organización. Particularmente una postura orientada a identificar las posibilidades ofrecidas por el trabajo antes que sus limitaciones, es decir, mucho más relacionada con un "hacerse cargo".

Respecto de la generación de Sentido. Entendiendo que el rol representa una intersección entre la organización y las personas, este tipo de intervención también provee espacios de reflexión y análisis del sistema donde participa el funcionario. En este nivel surgen elementos, que, a juicio de los participantes, determinan sus percepciones respecto a la calidad del trabajo. Algunos de estos elementos o factores son :

Rol de la Jefatura (Capacidad de liderazgo). La relación con las figuras de autoridad es un factor fundamental en la percepción de los funcionarios respecto a su trabajo. Está muy presente la necesidad de ser guiado, reconocido y valorado por los jefes, lo cual da cuenta del impacto de la cultura de dependencia que aún sigue presente en este tipo de organizaciones. 
97 Altos costos de transacción, es decir, baja productividad en términos de las coordinaciones al interior del equipo. Propio de una cultura burocrática construida sobre la base de la falta de confianza en el desempeño de los colegas. Esta situación es vivida como una gran limitación para desarrollarse laboralmente.

Escasez o ausencia de políticas de desarrollo, lo cual es recibido por los trabajadores como una falta de "interés" por parte de la institución para atender sus requerimientos de progreso. Deben ser entendidas como una limitación que impone el estatuto administrativo y que rige a todos los funcionarios del Estado, más que sólo una limitación de esta Institución en particular.

\section{Desafíos y oportunidades para una clínica del trabajo en Chile} culturalmente neoliberal porque se trata de una sociedad que ha vivido y valorado una gestión managerial en todos los ámbitos de la vida y en todos los sectores sociales, no sólo en el sector industrial. Además, y producto de que lleva casi 40 años en marcha, pareciera ser la única manera posible para hacer funcionar del sistema. Se suma a ello, que para países referentes de las Ciencias de la Gestión chilena (EEUU y Europa) es un modelo, pareciera pionero y moderno, dando lecciones a los que siempre han sido sus maestros.

100 En comparación con los años que preceden a la instalación de este modelo, Chile aparece ahora como un país más próspero, más desarrollado, más moderno, e incluso líder dentro de la región principalmente porque tiene menos problemas macroeconómicos que los vecinos latinoamericanos. La pobreza objetiva chilena de los años 70 es claramente mucho mayor que la que se tiene hoy. Nuestro país, está recibiendo la llegada de muchos trabajadores extranjeros, lo cual hacen pensar que nuestra localidad es un lugar de oportunidades para muchos trabajadores de América Latina.

101 Sin embargo, y como lo denuncia el movimiento social que estalla el 2019, detrás de todos estos supuestos, no hay una reflexión profunda en torno al endeudamiento de los trabajadores, no en bienes suntuarios, sino ligado a la alimentación, la vivienda, la educación y la salud de sus familias. La clase empresarial no crea industria, sino que administra, entre otros servicios, la electricidad, las aguas, las pensiones, los fondos de salud y las escuelas, enriqueciéndose de las necesidades básicas de la población.

Hasta el 2019, los trabajadores parecían haber aprendido y aceptado el silencio, desarrollando sus labores sin quejarse por el miedo a ser despedidos, sin grandes esperanzas en la sindicalización y con una obediencia ciega a sus jefaturas. El miedo a perder el empleo implica no sólo la pérdida de los ingresos, sino además la persecución del sistema financiero por no poder pagar las deudas que han contraído. Además, las formas de organización como el lean management, la evaluación por objetivos y los sistemas de bonos, implementadas en Chile durante casi 40 años, han convencido a los trabajadores que su salario depende de su esfuerzo individual y no de la cooperación entre pares. 
103 En ese sentido, las clínicas del trabajo no tuvieron un espacio sino hasta que la sociedad chilena y el mundo del trabajo, entraron en un momento crítico como el que evidenciamos hoy y que se manifiesta socialmente.

Hace menos de una década se nos abre paulatinamente un espacio en centros de trabajo del sector servicio porque existe un aumento de licencias médicas, de consumo de fármacos psiquiátricos asociados a la productividad y de depresiones y suicidios. Además, una nueva ley de Riesgos Psicosociales del trabajo da un lugar a esta perspectiva, porque la normativa obliga a medir el nivel de riesgos, pero además a generar grupos de discusión entre trabajadores que cumplen la misma función. La tarea de estos grupos es analizar las causas organizacionales de sufrimiento y las consecuencias individuales y colectivas de esta situación.

En un país neoliberal como Chile, la organización del management fortalece que los problemas son de rendimientos individuales y no problemas organizacionales. Los resultados de nuestro trabajo muestran la valoración positiva del dispositivo grupal. Este valor está dado por ciertos procesos que es interesante destacar.

106 El encuentro con el reconocimiento de su trabajo. En las tres intervenciones, el participante se encuentra con sus pares, con el reconocimiento de sus iguales. En general, las personas que trabajan en servicios se encuentran con los beneficiarios que son "clientes" y que exigen como consumidores, lejos de la "gratitud" que necesitan estos profesionales que están ayudando a la población.

Por otro lado, las jefaturas siempre exigen más y mejores resultados que no se pueden lograr sistemáticamente. Un año se pueden alcanzar las metas, pero el aumento no es sostenido en el tiempo. Es con los pares, hablando sobre temas de trabajo, que es posible encontrar el reconocimiento de la labor. Aquí aparece la noción de juicio de belleza (Dejours, 2009) de la tarea colectiva.

108 Abordar colectivamente el malestar individual. Trabajar grupalmente permite salir de la sensación de un problema individual a uno colectivo. Esto es valorado en profesiones tan distintas como enfermeras, ingenieros/as y profesores/as instalados en organizaciones públicas.

109 Los distintos trabajadores pueden expresar sus aprehensiones y angustias y pueden verlas vinculadas con la organización del trabajo y con los problemas de la sociedad chilena. Por otro lado, es posible realizar construcciones subjetivas que cuestionen los procesos narcisísticos en que las personas se sienten poderosas mientras logran los resultados demandados y que cuando están en falla, todo se desmorona y son puestas en el banquillo de los acusados de la institución. Los distintos trabajadores, en un espacio clínico del trabajo, observaron los vaivenes identitarios y subjetivos a los que están expuestos. Además, hay un espacio para la expresión de emociones (ira, frustración) distinta a las que la gestión empresarial demanda (satisfacción, alegría, empuje, ganas). Lo importante es que esos sentimientos no son por problemas entre personas, son problemas asociados a la organización del trabajo.

110 Reencontrarse con el sentido de la labor. Las tres experiencias se hacen cargo del sufrimiento y de los problemas para poder comprenderlos, abordarlos y tomar soluciones colectivas y coordinadas. En los tres casos, los compañeros de trabajo se ponen de acuerdo para mejorar la labor cotidiana. Estar pensado grupalmente no solo da alivio por constatar que no estamos solo, sino también de presionar a que las condiciones cambien de manera colectiva también. Frente a la culpabilización 
individual que el neoliberalismo ejerce sobre el trabajador, las clínicas del trabajo entregan otra mirada y muestran que ese sufrimiento está anclado en la forma de gestión. Esto hace a los ingenieros a identificar las posibilidades de desarrollo y capacitación, las enfermeras que sienten la necesidad de poner límites al trabajo y los profesores que identifican su capacidad de agencia real con los niños y niñas. Así, la idea agobiadora que todo depende de la voluntad del sujeto se termina volcando la mirada a la organización del trabajo.

111 En las experiencias presentadas se puede observar que se revitaliza una salud mental ligada a la capacidad de hacer bien el propio trabajo, de construir el hilo de la propia actividad, de mejorar lo que se hace y no con el fin instrumental, sino porque el trabajo está siempre dedicado a otros. En ese sentido, se trata de la salud mental cognitiva ligada al saber hacer de la tarea. Las

112 En todas las experiencias, la función del interventor es además la de un mediador con las figuras de autoridad de las organizaciones. Este lugar es más bien complejo, pues aparece como algo nuevo en un contexto donde hay ausencia de sindicatos en defensa de los trabajadores, ausencia de canales de comunicación y la dificultad cultural para abordar el conflicto. Este punto puede tener relación con la idea, heredada de dictadura, que el conflicto termina en violencia y además con las nociones funcionalistas propias del management que creen que el conflicto es un problema y no una condición de toda relación de trabajo.

Aparece así un primer desafío. ¿Cómo damos seguimiento a las intervenciones? ¿Cómo usamos el espacio de este "tercero que ayuda" a las organizaciones que están focalizadas en el rendimiento? ¿Qué rol macro y micro político cumplimos? Y luego, un desafío mayor, que hace alusión al destino político que damos al sufrimiento. Estamos ante una la tarea de articular el sufrimiento como una forma de pensar la organización y especialmente la gestión del Estado. Romper la asociación de modernidad con explotación y dar espacio a una nueva forma de defender nuestros derechos.

114 Ya llevamos casi 40 años de neoliberalismo, ya dimos la vuelta y ya mostramos que no se destruye la comunidad y tiene costos en la subjetividad. Ahora tenemos la tarea de hacer algo nuevo y los trabajadores señalan que tenemos un rol posible. Hoy, más que nunca en Chile, porque Chile despertó y no quiere más neoliberalismo.

\section{BIBLIOGRAFÍA}

Acuña, E., \& Sanfuentes, M. (2008). Coaching : Análisis del rol organizacional. Santiago de Chile : Editorial Universitaria.

Acuña, E., \& Sanfuentes, M. (2013). Métodos socioanalíticos : para la gestión y el cambio en organizaciones. Santiago de Chile : Editorial Universitaria.

Araujo, K. (2016). El miedo a los subordinados. Una teoría de la autoridad. Santiago de Chile : LOM. 
Ansoleaga, E., Dávila, A., Jara, M., \& Lucero, C. (2014). La importancia de lo colectivo en la organización del trabajo : la experiencia de una clínica psicosocial del trabajo en un servicio público de oncología pediátrica de alta complejidad en Chile. In A. M. Mendes, R. Moraes \& A. Merlo (Dirs.), Sufrimiento en el Trabajo : perspectivas políticas y clínicas (pp. 41-44). Curitiba : Editora Juruá.

Barus-Michel J. (2004). Souffrance, sens et croyance. L'effet thérapeutique. Toulouse : Érès.

Bauman, Z. (2013). Liquid modernity. Cambridge: John Wiley \& Sons.

Boltanski, L., \& Chiapello, E. (1999). Le nouvel esprit du capitalisme. Paris : Gallimard.

Clot, Y., \& Lhuilier D., (2010). Agir en clinique du travail. Toulouse : Érès.

de Gaulejac, D. (2000). Relatos de Vida e historia social. In E. Taracena (Dir.), La sociología clínica. Una propuesta de trabajo que interroga las barreras disciplinarias (pp. 53-86). México : UAM.

de Gaulejac, V. (2005). La société malade de la gestion. Paris : Seuil.

de Gaulejac, V. (2011). Travail, les raisons de la colère. Paris : Seuil.

de Gaulejac, V., Giust-Desprairies, F., \& Massa, A. (2013). La recherche clinique en sciences sociales. Toulouse : Érès.

de Gaulejac, V., \& Hanique, F. (2014). Le capitalisme paradoxant. Paris : Seuil.

de Gaulejac, V., \& Guerrero, P. (2017). La sociedad parajoxante : un sistema que vuelve loco. In H. Foladori \& P. Guerrero (Dirs.), Malestar en el Trabajo : Desarrollo e Intervenciones (pp. 8-33). Santiago de Chile : LOM Ediciones.

De la Garza Toledo, E., \& Neffa, J. (2010). Trabajo y modelos productivos en América Latina : Argentina Brasil, Colombia, México, y Venezuela luego de las crisis del modo de desarrollo neoliberal. Buenos Aires : Consejo Latinoamericano de Ciencias Sociales - CLACSO.

Dejours, C. (2009). Travail vivant. Tome 2 : Travail et émancipation. Paris : Payot.

Gárate, M. (2012). La revolución capitalista de Chile :(1973 - 2003). Santiago de Chile : Ediciones Universidad Alberto Hurtado.

Gastal de Castro, F., \& Guerrero, P. (2013). L'organidrame et possibilités de changement : deux réflexions théorique-méthodologique sur un dispositif d'intervention en conflits socio-clinique. In F. Giust-Desprairies, A. Massa \& V. de Gaulejac (Dirs.), La recherche clinique en sciences sociales (pp 219-236). Toulouse : Érès.

Guerrero, P. (2008). La aproximación clínica del trabajo : una posibilidad de intervenir e investigar la identidad profesional de los docentes de zonas vulnerables. Psiquiatría y Salud Mental, 25(1-2), 92-104.

Guerrero, P. (2017). Cooperación y Sociología clínica : una propuesta de formación de profesores. In N. Ruiz \& H. Foladori (Dirs.), Cooperación en los Vínculos Grupales e Institucionales (pp. 55-73). Santiago de Chile : Editorial Universitaria.

Honneth, A. (2006). La société du mépris : vers une nouvelle théorie critique. Paris : La Découverte.

Huneeus, C. (2016). El régimen de Pinochet. Santiago de Chile : Taurus.

Kocyba, H., \& Renault, D. (2007). Reconnaissance, subjectivisation, singularité. Travailler, (2), 103-118.

Lhuilier, D. (2006). Cliniques du travail. Toulouse : Érès.

OECD (2015). Estudios económicos de la OECD CHILE. Paris : OECD. 
Offe, K. (1997). ¿Qué crisis ? Retos y transformaciones de la sociedad del trabajo. Donostia : Tercera Prensa.

Pichon-Rivière, E. (1985). El proceso grupal : del psicoanálisis a la psicología social. Buenos Aires : Editorial Nueva Visión.

PNUD (2017). Chile en 20 años. Un recorrido a través de los Informes sobre Desarrollo Humano. Santiago de Chile : Programa de Naciones Unidad para el Desarrollo Chile https://www.undp.org/content/ dam/chile/docs/desarrollohumano/undp_cl_idh_Chile-en-veinte-anos-doc.pdf

Priestley, M., Biesta, G., \& Robinson, S. (2015). Teacher agency: an ecological approach. Londres : Bloomsbury Academic.

Ramos, C. (2009). La transformación de la empresa chilena : una modernización desbalanceada. Santiago de Chile : Ediciones Universidad Alberto Hurtado.

Rosa, H. (2010). Accélération : une critique sociale du temps. Paris : La Découverte.

Sisto, V. (2012). Identidades desafiadas : individualización, managerialismo y trabajo docente en el Chile actual. Psykhe (Santiago), 21(2), 35-46.

Stecher, A. (2015). La empresa flexible como dispositivo de gobierno. Aportes de la Analítica de la Gubernamentalidad al estudio de las subjetividades laborales en América Latina. Universitas Psychologica, 14(5), 179-194.

Stecher, A., \& Godoy, L. (2014). Transformaciones del trabajo, subjetividad e identidades. Lecturas psicosociales desde Chile y América Latina. Santiago de Chile : RIL Editores.

UNESCO (2017). Rendir cuentas en el ámbito de la educación : cumplir nuestros compromisos. Informe de Seguimiento de la Educación en el Mundo. Paris : UNESCO. http://unesdoc.unesco.org/images/ 0025/002595/259593s.pdf.

Viviers, S., Boulet, J., \& Hamelin, G. (2018). Santé psychologique et souffrance identitaire de métier : résultats d'une enquête sur l'expérience du travail de C.O. en milieu scolaire. L'Orientation , 8(1), 10-14.

Voswinkel S., Gernet I., \& Renault E. (2007). L'admiration sans appréciation. Les paradoxes de la double reconnaissance du travail subjectivisé. Travailler, 2(18) 59-87.

Zabala, X., Guerrero, P., \& Besoain, C. (2017). Clínicas del Trabajo. Teorías e intervenciones. Santiago de Chile : Ediciones Universidad Alberto Hurtado.

\section{NOTAS}

1. Grafiti encontrado el 2019

2. La palabra managerial no existe en español, pero la usaremos para referirnos a la organización que viene del management y que Vincent de Gaulejac, ha caracterizado en el libro "Société malade de la gestion" (2005).

3. En este artículo y en el libro "Clínicas del Trabajo: Nociones y perspectivas" que se publicó el 2017 bajo la coordinación de Ximena Zabala, Patricia Guerrero y Carolina Besoain en las Ediciones Alberto Hurtado.

4. MINEDUC (2015). Diversificación de la enseñanza. Decreto $n^{\circ}$ 83/2015: Aprueba criterios y orientaciones de adecuación curricular para estudiantes con necesidades educativas especiales de educación parvularia y educación básica.

5. MINEDUC (2017) Orientaciones sobre estrategias diversificadas de enseñanza para educación básica, en el marco del decreto 83/2015. 
6. Núcleo Milenio Autoridad y Asimetrías de Poder, liderado por Kathya Araujo y Antonio Stecher. Ver http://www.iniciativamilenio.cl/autoridad/

\section{RESÚMENES}

El artículo se propone problematizar el proceso de transformaciones del trabajo contemporáneo en Chile. Se describen tres experiencias desde las clínicas del trabajo (educacional, psicosocial y socioanálisis) en diferentes escenarios laborales. A su vez, se realiza una reflexión sobre la pertinencia de la clínica del trabajo como forma de intervención para contribuir a la comprensión de los procesos subjetivos de los trabajadores, en su dimensión individual, grupal y política.

O artigo propõe-se problematizar o processo de transformações do trabalho contemporâneo no Chile. Três experiências são descritas a partir de estudos desenvolvidas na perspetiva das clínicas do trabalho (educacional, psicossocial e socioanálise) em diferentes cenários de trabalho. A seguir, faz-se uma reflexão sobre a relevância da abordagem da clínica do trabalho como forma de intervenção que contribui para a compreensão dos processos subjetivos dos trabalhadores, nas suas dimensões individual, grupal e política.

L'article propose une problématisation du processus de transformation du travail contemporain au Chili. Trois expériences sont décrites au départ d'études menées selon la perspective des cliniques de travail (éducatives, psychosociales et socio-analytiques) dans différents scénarios de travail. Une réflexion est menée ensuite à propos de la pertinence de l'approche de la clinique du travail, définie en tant que mode d'intervention contribuant à la compréhension des processus subjectifs des travailleurs, dans leur dimension individuelle, de groupe et politique.

The paper aims at problematizing the process of contemporary work transformations in Chile. Three experiences are described from a clinical point of view (pedagogical, psychosocial and socio-analytical) in different work scenarios. Then, it reflects on the relevance of the clinical approach as a form of intervention, which contributes to the understanding of the workers' subjective processes, in their individual, group and political dimensions.

\section{ÍNDICE}

Mots-clés: transformations du travail contemporain, sujet social, cliniques du travail, clinique psychosociale du travail, socioanalyse

Palabras claves: transformaciones del trabajo contemporáneo, sujeto social, clínicas del trabajo, clínica psicosocial del trabajo, socioanálisis

Keywords: contemporary work transformations, social subject, work clinics, psychosocial clinical work, socio-analysis

Palavras-chave: transformações do trabalho contemporâneo, sujeito social, clínicas do trabalho, clínica psicossocial do trabalho, socioanálise 


\section{AUTORES}

\section{PATRICIA GUERRERO MORALES}

Facultad de Educación, Pontificia Universidad Católica de Chile ; Vicuña Mackena 4680, Macul, Santiago de Chile, Chile ; pguerrem@uc.cl

\section{MANUEL GÁRATE CHATEU}

Instituto de Historia, Pontificia Universidad Católica de Chile ; Vicuña Mackena 4680, Macul, Santiago de Chile, Chile ; mgarate@uc.cl

\section{ELISA ANSOLEAGA IMAS}

Facultad de Psicología, Universidad Diego Portales ; Vergara 275, Santiago de Chile, Chile ; elisa.ansoleaga@gmail.com

\section{J. MARCELO BALBOA GALLARDO}

Universidad Laval ; 858, Isidore Garon, app 1, Quebec, Canada ; marcelobalboa.psi@gmail.com

\section{JOSÉ TOMAS SAFFIRIO MEZZANO}

Facultad de Economía y Negocios, Universidad de Chile ; Eliodoro Yañez 2972 of.605, Providencia, Santiago de Chile, Chile ; saffirio@sintoniza.cl

\section{ALEJANDRA NUÑEZ CARBULLANCAUTOR}

Facultad Economía y Negocios, Universidad de Chile ; Eliodoro Yañez 2972 of.605, Providencia, Santiago de Chile, Chile ; anunez@sintoniza.cl

\section{VALENTINA URRUTIA FUENZALIDA}

Consultora Educación y Trabajo ; Vicuña Mackena 4680, Macul, Santiago de Chile, Chile ; valentinaurrutiafuenzalida@gmail.com 